\title{
Possibility of insulator to superconductor phase transition
}

\author{
B. K. Chakraverty \\ Groupe des Transitions de Phases, C.N.R.S., \\ B.P. 166, 38042 Grenoble Cedex, France \\ (Reçu le 20 novembre 1978, accepté le 9 janvier 1979)
}

\begin{abstract}
Résumé. - On propose qu'un système avec un fort couplage électrons-phonons est un isolant bipolaronique au-delà d'une constante de couplage critique. Ceci donne la possibilité d'une transition de phase d'un état supraconducteur à l'état isolant à $T=0$ quand on varie la constante de couplage.
\end{abstract}

\begin{abstract}
A phase diagram is postulated where the ground-state of a strongly coupled electron-phonon system is a bipolaronic insulator beyond a critical coupling strength. This opens up the possibility of phase transition from a superconductor to insulator at $T=0$, as the coupling is varied.
\end{abstract}

It is generally believed that a superconductor is metallic in its normal state. We propose that the complete phase diagram must contain a branch where the superconducting state becomes insulating above the transition temperature $T_{\mathrm{c}}$.

In a series of recent papers the existence of a bipolaronic ground-state in a great variety of non-metallic transition metal compounds has been both postulated [1] and demonstrated [2]. These bipolarons can be thought of as localized Cooper-pairs (a pair of upspin down-spin electrons on near-neighbour sites) that are stable as long as the deformation-induced mutual attraction term $\frac{g_{0}^{2}}{M \omega_{0}^{2}}$ exceeds the mutual Coulomb repulsion $v$, where $g_{0}$ is an optical electronphonon coupling constant, $M$ is the atomic mass and $\omega_{0}$ is an optical frequency. These bipolarons stand exactly in the same relationship to B.C.S. superconducting pairs as does a localized electron to an itinerant one.

The analogy between the two kinds of electron pairs is very close indeed and is the subject of a fuller paper [3] under preparation. In order to see this analogy simply, we may naturally ask the question as to what happens to a B.C.S. pair as we increase the electron-phonon coupling. The McMillan formulation [4] of the B.C.S. theory of superconductivity gives us, in the strong-coupling limit $T_{\mathrm{c}}$ as a function of $\lambda$, the dimensionless electron-phonon coupling constant which is given by

$$
\lambda=\frac{N(0)\left\langle g^{2}\right\rangle}{M\left\langle\omega^{2}\right\rangle}
$$

where $N(0)$ is the density of electronic states of one spin at the Fermi level/eV-atom.

$\left\langle g^{2}\right\rangle$ is the electron-phonon coupling constant averaged over the Fermi surface,

and $\left\langle\omega^{2}\right\rangle \ldots$ some averaged acoustic phononfrequency squared.

We may note that $\frac{g_{0}^{2}}{M \omega_{0}^{2}}$ is the maximum binding energy of a bipolaron, if the Coulomb repulsion term is ignored and a $\lambda_{\max }$ corresponding to some maximum electron-phonon coupling constant for bipolaron formation can be defined. Here both $g_{0}$ and $\omega_{0}$ correspond to optical phonons, especially the $k=0$ mode. As an example, a value of $\lambda_{\max }$ can be obtained for the bipolarons in $\mathrm{Ti}_{4} \mathrm{O}_{7}$ for which we have the most extensive data. Using a binding energy of bipolaron formation of $0.6 \mathrm{eV}$ as equal to $\frac{g_{0}^{2}}{M \omega_{0}^{2}}$ with $N(0)$ equal to 5 states/eV-atom as obtained from the Pauli susceptibility measurements, we obtain a $\lambda_{\max }=3.0$, which is much larger than that observed experimentally for all known superconducting materials. We can use the strong-coupling parameter $\lambda$ to describe two limiting behaviours of the ground-state of a strongly coupled electron-phonon system.

(a) In the limit $\lambda<1$, the McMillan expression for the superconducting transition temperature $T_{\mathrm{c}}$ reduces to the B.C.S. expression

$$
T_{\mathrm{c}} \sim\langle\omega\rangle \exp -\left|\frac{1+\lambda}{\lambda}\right| .
$$


Although this means that even for an infinitesimally small $\lambda, T_{\mathrm{c}}$ will be non-zero, for all real practical purposes $T_{\mathrm{c}}$ is very small below a $\lambda \sim 0.1$ (e.g, $\mathrm{Na}$ would be superconducting with a $T_{\mathrm{c}} \sim 10^{-3} \mathrm{~K}$ !) and we can use a critical value of $\lambda$, as a threshold for the appearance of superconductivity. For most metals and alloys that become superconducting, $\lambda \sim 0.1$ to 1 [4]. The full strong-coupling McMillan expression for $T_{\mathrm{c}}$ shows saturation of $T_{\mathrm{c}}$ beyond $\lambda \sim 2$ (the socalled $\lambda=2$ limit for maximum temperature superconductivity). Allen and Dynes [6] have shown that this $T_{\mathrm{c}}$ maximum is an artifact of the McMillan expression, and in reality $T_{\mathrm{c}}$ should continue to increase with $T_{\mathrm{c}} \sim \sqrt{\lambda}$ when $\lambda$ is as large as 10 or beyond. We do not think that this has any physical meaning, and that, on the contrary, some sort of saturation and eventual decrease of $T_{\mathrm{c}}$ with $\lambda$ must occur before that.

This merely underlines the necessity that in the McMillan-Allen-Dynes formulation the renormalization of the $\left\langle\omega^{2}\right\rangle_{\text {av }}$ must be included in such a way that in the strong coupling limit $\left\langle\omega^{2}\right\rangle_{\mathrm{av}} \rightarrow 0$, as $\lambda \rightarrow \lambda_{2}$ leading to a $T_{\mathrm{c}} \rightarrow 0$. The point $\lambda=\lambda_{2}$ is precisely where the local deformation is static rather than virtual.

(b) We know that in the large $\lambda$ limit, bipolaron formation occurs. For a simple square density of states of bandwidth $W$ and a half-filled band, the metallic state is unstable to bipolaron formation, if the binding energy $E_{\mathrm{b}}$ of bipolarons $\left(E_{\mathrm{b}}=\frac{g_{0}^{2}}{M \omega_{0}^{2}}\right)$ exceeds $W / 2$. This gives us a $\lambda_{2}=2.5$ as the second limit on the electron-phonon coupling constant at which the metallic state becomes insulating at $T=0$. We can thus postulate that at a second critical electronphonon coupling constant $\lambda=\lambda_{2}, \lambda_{2}>\lambda_{1}$ the ground-state will be bipolaronic.

(c) At $T \neq 0$, the insulating ground-state of the bipolarons eventually transforms to a metallic state as has been shown by the extensive experimental work of C. Schlenker [7] and her group.

From the considerations $(a),(b)$ and $(c)$, the compo-

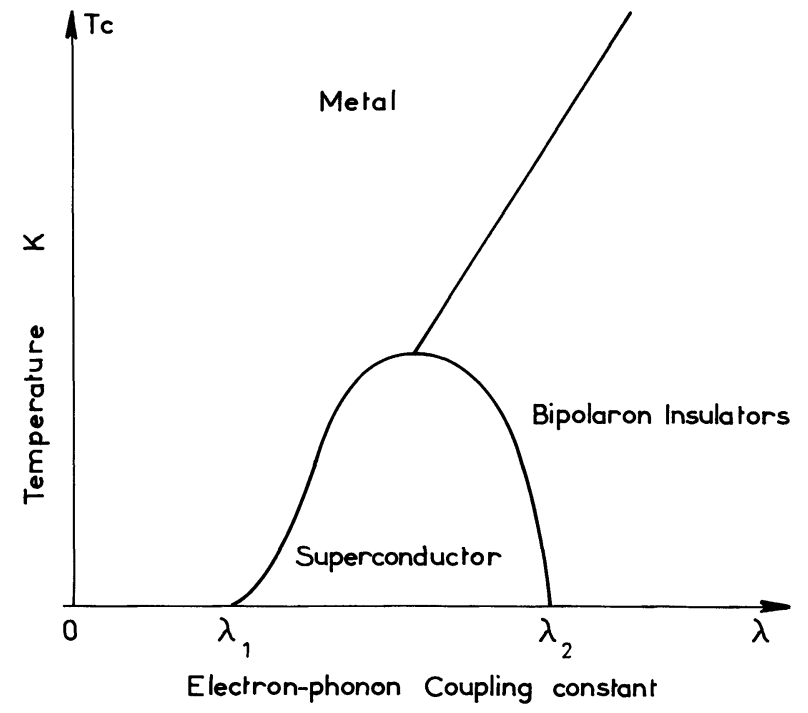

Fig. 1. - Phase diagram as a function of electron-phonon coupling strength.

site phase-diagram as a function of the electronphonon coupling parameter $\lambda$ must look like figure 1 . It is thus seen that a superconductor to insulator phase transition must inevitably occur.

Several points are to be noted. At $T=0$, this transition, we believe, will occur abruptly and will be first order. The three phases cannot coexist at the point $\lambda_{2}$ at $T=0$ because in the presence of any attractive pairing interaction between two electrons the eigen-states must be paired. The decrease of the superconducting temperature below a certain $\lambda$ really reflects the instability of the lattice towards covalent bond formation (crystalline Be with a $T_{\mathrm{c}} \sim 0.3 \mathrm{~K}$ almost succeeds being a semiconductor like its neighbour B). This tendency of transition towards covalent non-metallic structure with increased $\lambda$ was already pointed out by Anderson and Cohen [8].

Dr. Julius Ranninger of this laboratory raised as many objections to this paper as possible, but graciously conceded to me the phase diagram.

\section{References}

[1] Chakraverty, B. K. and Schlenker, C., J. Physique Colloq. 37 (1976) C4-353.

[2] Lakkis, S., Schlenker, C., Chakraverty, B. K., Buder, R. and Marezio, M., Phys. Rev. B 14 (1976) 1429.

Chakraverty, B. K., Sienko, J. M. and Bonnerot, J., Phys. Rev. B 17 (1978) 10, 3781

[3] Chakraverty, B. K., under preparation.

[4] McMillan, W. L., Phys. Rev. 167 (1968) 331.

[5] Appel, J., Solid State Phys. 21 (1968) 193, eds. Seitz and Turnbull.
[6] Allen, P. B. and Dynes, R. C., Phys. Rev. B 12 (1975) 905.

[7] LaKKIS, S., Thèse d'Etat, Université de Grenoble (1976). Ahmed, S., Thèse de $3^{\mathrm{e}}$ Cycle, Université de Grenoble (1977). Gourmala, M., Thèse de $3^{\mathrm{e}}$ Cycle, Université de Grenoble (1978).

[8] Anderson, P. W. and Cohen, M. L., Superconductivity in $d$ and f-band metals, ed. D. H. Douglass (American Institute of Physics) 1972, p. 24. 